\title{
LES PARAMOS D'ESPAGNE ET PARAME (BRETAGNE)
}

Paul Quentel

Il y a longtemps que l'on a revelé l'interérêt linguistique et historique des Páramos du nord de l'Espagne. On en compte deux dans la province de Burgos, deux à Palencia, deux dans les Asturies, deux dans le León, deux à Lugo, deux à Pontevedra, quatre à la Corogne. Ils sont attestés dès l'époque romaine. Ptolémée et d'autres auteurs mentionnent Segontia Paramica. En 458 il est fait état d'une bataille entre Goths et Suèves "in campo Paramo". La forme Paramio apparaît dans un document portugais de $1050^{1}$. Géographiquement, certains ouvrages qualifient les Páramos de "zones désertiques". Mais ce n'est pas là leur caractéristique essentielle: ce sont toujours des lieux élevés, des "plateaux". La question qui se pose, au sujet de ces noms, est la suivante: à quel peuple faut-il les attribuer?

Ici, les avis diffèrent. C'est un mot clairement indo-européen, estime J. Pokorny, qui fait valoir qu'on ne peut séparer ce nom du sanscrit paramah "le plus élevé". Mais, comme Páramo a conservé le $\mathrm{p}$ initial, il serait pré-celtique ${ }^{2}$. C'est aussi l'opinion d'Antonio Tovar $^{3}$. Pour R. Menéndez Pidal, qui souligne surtout dans ce nom la finale -amo, comparée par lui avec le suffixe- amo de légamo, préstamo, balamo, etc..., il s'agit d'une "voz ibérica"4 . J. Hub-

1 J. Corominas, Diccionario crítico etim. de la lengua castellana, tome 3, Berne 1954, s.v.

2 J. Pokorny, Zeitschrift für celt. Philo., XXI, 150; Féil-Sgríbinn Eoin Mic Néill, Dublín 1940, 239.

3 A.T., Estudios sobre las primitivas lenguas hispánicas, Buenos Aires, 1949, 196, n.I.; Investigaciones sobre la onomástica de la Hispania prerromana, Onoma, 2, $1951,36$.

4 R.M.P., Toponimia prerromana Hispana, Madrid 1952, 69. 


\section{PAUL QUENTEL}

schmid, quant à lui, classe Páramo, comme Palencia, dans une catégorie qu'il appelle "para-celtique" et qu'il attribue à un peuple arrivé en Espagne en même temps que les Celtes ${ }^{5}$. Harri Meier, parle, à propos de ces thèses sur la haute antiquité de Páramo, de "mirage"6. Le toponymiste belge A. Carnoy enseigne que Páramo "est un nom hispanique, nettement indo-européen, mais qui ne peut être celtique, à cause du p-" pour J. Corominas: ou bien il s'agit d'une forme ultra-archaïque du celtique, ou bien c'est un mot relevant d'une autre famille indoeuropéenne" 8 . Ulrich Schmoll voit dans Paramus, un "Lehnwort aus Hispanien, spa. Páramo, 'Hochebene"

Ces travaux ne citent pas de formes de Paramus au nord des Pyrénées. Cependant, en Aquitania prima, Joshua Whatmough relève une inscription du Corpus des inscriptions latines "In Parami aequore, C.I.L., 2. 2660"10 , qu'il considère comme ibère, non sans hésiter toutefois, car, fait-il remarquer après Meyer-Lubke, en ibère aussi le p-est quelque peu anormal ${ }^{11}$.

La question des Páramos d'Espagne se trouve singulièrement éclairée par la présence, sur la côte nord de la Bretagne, à quelque 700 kilométres des Pyrénées, d'un toponyme qui est le même: il s'agit de Paramé, ancienne paroisse, ancienne commune, mais qui a fusionné avec Saint Malo il y a dix ans; et ceci d'autant plus que, à côté de Paramé, un autre nom apporte, lui aussi, sa contribution à l'histoire de Notre toponyme.

5 J.H., Toponimia prerromana, Enciclopedia lingüística hispánica, I, 1959, 447-493; p. 132. l'auteur définit sa pensée comme suit: "No se puede hablar de un pueblo precelta con p-, sino más bien de un publo con p- que llegó a Hispania con los Celtas". Rappelons que P. Bosch-Gimpera parle de deux invasions celtiques en Hispanie.

6 Harri Meier, Kritische Betrachtungen zur romanischen Substrate-tymologie, Romanische Forschungen, 64, 1952, I-42. Roca n'est nullement latín; cf J. Corominas, Zeitschrift für celt. Phil., XXV, 20 sq qui cite un composé de roca avec le préfixe celtique bien connu vero-. J'ai relevé moi-même son antonyme en Bretagne, goroc'h "petite roche".

7 A.C. Dictionnaire etym, du proto-européen, Louvain 1955139.

8 J.C., 1.c.

9 U.S. Die Sprachen der vorkeltischen Indogermanen Hispaniens und das Keltiberische, Wiesbaden 1955, 44.

10 J.W., The dialects of ancient Gaul, Harvard 1970, 428.

11 Ibid, 466. 


\section{LES PARAMOS D'ESPAGNE ET PARAME}

Les formes anciennes, ici, sont habituellement Paramus et Paramé. On trouve aussi les variantes "Ecclesia de Passu ramato" en 1329, "Pas ramé" en 1455, Parrame en $1419^{12}$. Passu ramato et Pas ramé sont evidemment l'oeuvre de sribes férus d'etymologie: les noms en Pas (type: Pas de l'oie, Pas au boeuf, Pas, suivi d'un nom de personne, etc...) sont nombreux dans le voisinage de Saint Malo, au sens de "passage"13. Mais il n'y a pas à s'attarder sur ces interprétations: la forme actuelle, Paramé, est ancienne. Géographiquement, Paramé désigne une localité située sur la partie ouest d'un plateau de plus de dix kilomètres, qui s'étend jusqu'à Cancale. A tous égards donc, le toponyme breton est le même que celui d'Espagne.

Les conséquences de cette similitude sont importantes. Ici, en effect, on ne peut parler d'Ibères. S'il a éxisté, d'autre part, dans cette région, une langue non-indoeuropéenne quelconque, ses survivances en toponymie sont bien maigres. Et, en matière de langue indo-européenne ancienne, seul le celtique est très largement représenté.

Avant de tirer des conclusions plus précises, il faut relever l'existence, à côté de Paramé, d'un autre toponyme, Rotheneuf. Ce nom, en 1493, est Roteneuf, en 1541 Rosteneuc, en 1558 Roteneuc $^{14}$. La forme de 1541 est parfaitement claire: bien que relevée postérieurement à celle de 1493, c'est d'elle que découle Roteneuc, puis Roteneuf. Le premier élément est Ros, bien connu en Irlande, Grande et Petite Bretagne, au sens de "promontoire, plateau, côte, butte, etc...". Dans la toponymie de la région de Saint Malo, où ce nom est plus souvent orthographié roz, la prononciation est "ro" (Cf Roz sur Couesnon, Roz Landrieux). Ros remonte à un celtique rostro (cf. Sanscrit prastha, plateau). Mais, en fait, rostro suppose lui-même un archaïque prosto. Nous constatons donc l'absence habituelle du p- initial dans un mot de sens identique à celui de Paramé, comme cela est le cas, par exemple, dans le gaulois Aremorica, où le premier élément correspond au latin $\mathrm{Para}^{15}$. Et, puisque Aremorica vient

12 Bossard, Diction. top. d'Ille et V., ms $5 \mathrm{Fg} \mathrm{2,} \mathrm{Archives} \mathrm{d'I.} \mathrm{et} \mathrm{V.,} \mathrm{Rennes,} \mathrm{s.v.}$ Paramé.

13 Sur Pas dans ce sens, cf Hermann Grohler, Uber Ursprung und Bedeutung der franz. Ortsnamen, Heidelberg, 1933, 145, s.v. Passus.

14 Bossard, o.c. s.v. Rothenenuf.

15 Les autres élements composant Rotheneuf sont Ten (n), abrupt, raide, et la désinence -euc, Pour l'évolution de Teneuc en Teneuf, cf Lesteneuf, en Plénée-Jugon (C. du N.) 


\section{PAUL QUENTEL}

d'être mentionné, on peut y relever aussi la présence de la désinence d'adjectif-ica, bien connu en celtique, et en rapprocher celle de (Segontia) Paramica que donnet Corominas et Schmoll.

De ces remarques, on peut tirer plusieurs conclusions.

La première, c'est que, malgrè la chute du p- initial en celtique, de façon générale, celle-ci n'a eu lieu qu'au cours de l'évolution propre au celtique. En d'autres termes, le celtique archaïque peut avoir conservé le P- initial, au moins sur le continent. En Espagne, il n'y a pas lieu de faire objection à la celticité de plou, pleu, que l'on trouve en composition dans Complutum, pour désigner un confluent $^{16}$ ou encore à celle de Pelendones ou de Bletisama (où Blet est pour Plet, cf grec $\pi \lambda \propto_{\tau} \propto_{c} \propto 1$ ' "très plat") que mentionne A. Tovar $^{17}$ ) ni, en France, à la celticité du nom des Parisii ou celui des Pictavi. En Allemagne, il n'y a pas lieu, non plus, de classer automatiquement comme "pré-celtiques", et pour la même raison, des noms de cours d'eau comme Pall, Prims (ancien Primantia), Pallien ${ }^{18}$ : ce ne sont là que quelques exemples entre beaucoup d'autres.

La seconde, c'est que l'expression "ultra archaïque" qu'emploie Corominas paraît tout à fait appropriée. Depuis les environs de 1925 surtout on fixe couramment l'établissement des Celtes dans 1'Europe de l'ouest au VI é, voire au Vé siécle avant J.C. Dans un autre travail, en cours de publication, il sera montré que, à une époque, antérieure de plusieurs millénaires à celle-là, on constate déjà la présence des Celtes à l'extrême ouest de l'Europe. Les contacts dont fait état l'Ora Maritima d'Avienus, entre le N.O. de la péninsule hispanique et la Bretagne (actuelle), qui sont corroborés par l'archéologie, sont vraisemblablement, en fait, l'histoire de la celtisation de l'Europe de l'Ouest, bien antérieurement à la civilisation dite de Hallstadt. Ce n'est pas un pur hasard que de retrouver, dans le N.O. de l'Espagne, d'une part, et en Bretagne, d'autre part, un même toponyme celtique "ultra archaîque".

"la cour d'accès difficile" (les: cour). Ten (n) se retrouve dans Tinteniac (Tin, var. de Din, fort) qui désigne un gros bourg entre Rennes et Saint Malo.

Cf. J. Corominas, o.c.

17 A. Tovar, Estudios, o.c.

18 Comme le fait Hans Kuhn, Die Nordgrenze der keltischen Ortsnamen in Westdeutscland, Beiträge zur Namenforschung, Bd 3, 1968, Heft, 4, 314. 